Eurostudia

\title{
Grenzgänger in der Literatur
}

\section{David Simo}

Volume 7, numéro 1-2, 2011

Deutschland und Europa : Grenzen und Grenzgänge(r)

L'Allemagne et l'Europe : frontières et passeurs

URI : https://id.erudit.org/iderudit/1015019ar

DOI : https://doi.org/10.7202/1015019ar

Aller au sommaire du numéro

Éditeur(s)

Le Centre canadien d'études allemandes et européennes

ISSN

1718-8946 (numérique)

Découvrir la revue

Citer cet article

Simo, D. (2011). Grenzgänger in der Literatur. Eurostudia, 7(1-2), 175-180.

https://doi.org/10.7202/1015019ar

Tous droits réservés ( Le Centre canadien d'études allemandes et européennes, 2012
Ce document est protégé par la loi sur le droit d'auteur. L'utilisation des services d'Érudit (y compris la reproduction) est assujettie à sa politique d'utilisation que vous pouvez consulter en ligne.

https://apropos.erudit.org/fr/usagers/politique-dutilisation/
Cet article est diffusé et préservé par Érudit.

Érudit est un consortium interuniversitaire sans but lucratif composé de l’Université de Montréal, l'Université Laval et l'Université du Québec à Montréal. Il a pour mission la promotion et la valorisation de la recherche. https://www.erudit.org/fr/ 
David Simo

Université de Yaoundé 1

$\mathrm{D}$ ie Literatur stellt eine Institution dar, in der Grenzen, Grenzziehung, aber auch Grenzüberschreitungen sowohl thematisch, als auch konzeptionell und diskursiv eine wichtige Rolle spielen. Gerade in Europa pendelt ihr Selbstverständnis zwischen Universalismus und Partikularismus, Nationalisierungstendenzen und Transnationalisierungsperspektive.

\section{LITERATUR UND NATION ODER VERORTUNGSTENDENZEN DER LITERATUR}

Kafka meinte zwischen der Literatur einer großen und der einer kleinen Nation unterscheiden zu können. ${ }^{1}$ Als kleine Nation bezeichnete er eine religiöse oder ethnische Gemeinschaft, die unter einer repressiven Herrschaft lebt. Hier konstituiert sich das kollektive Gedächtnis als eine Widerstandsstrategie und als Mittel der Befreiung. Das Bewusstsein, eine Gruppe $\mathrm{zu}$ sein, ist alles andere als selbstverständlich. Das Bewusstsein, eine Gemeinschaft zu bilden, setzt eine diskursive Praxis voraus. Nur dadurch, dass man davon spricht, dass man eine Gemeinschaft bildet und sich entsprechend verhält, nimmt man sich als Gemeinschaft wahr. Die Wirklichkeit der Gemeinschaft muss erst sprachlich konstruiert werden, um eine Wirklichkeit an und für sich zu werden. Und die Literatur spielt dabei eine entscheidende Rolle, so zum Beispiel bei den historischen Fällen, die Kafka behandelt, nämlich dem Fall der tschechischen Minderheit in Prag und der jüdischen Minderheit in Warschau. Hier bildet die Literatur ein gemeinsames Gedächtnis sowie einen gemeinsamen geistigen Raum.

Aber nicht nur kleine und bedrohte Nationen rekurrieren auf die Literatur als Ort der Vergewisserung und Konsolidierung des Nationalbewusstseins. Die Geschichte zeigt, wie sehr auch starke Nationen die Literatur als Mittel der Darstellung und als Nachweis ihrer Größe verwenden.

Die indische Literaturwissenschaftlerin Viswanathan hat hierzu eine höchst interessante Studie durchgeführt. ${ }^{2}$ Darin untersucht sie, wie die Engländer das Studium der englischen Literatur in Indien im 19. Jahrhundert als Herrschaftsmittel einführten, und zwar nicht nur, weil diese ethische Gedanken enthielt, die den kolonisierten einheimischen Völkern moralische Werte und Respekt gegenüber den

\footnotetext{
${ }^{1}$ Franz Kafka, Tagebücher 1910-1923 (Frankfurt/M: Fischer Taschenbuch, 1973).

${ }^{2}$ Gauri Viswanathan, "The Beginning of English Literary Study in British India," in The Post-colonial Studies Reader, hg. Bill Ashcroft, Gareth Griffith, Helen Tiffin (London und New York: Routledge, 1995).
} 
Gesetzen beibringen sollten, sondern auch, um der geistigen Größe und Überlegenheit Englands Ausdruck zu verleihen. Sie sollte den Einheimischen Respekt und Bewunderung einflößen und sie veranlassen, die Herrschaft Englands als natürlich und notwendig anzuerkennen.

Während die Literatur eines Landes im Ausland als Herrschaftsmittel verwendet wird, wird sie im Land selber zu einem Objekt, um das herum Kohäsion und Homogenität hergestellt wird. Für die Nachbarländer wird sie zum Ort der Sichtbarkeit der Alterität der Nationalidentität.

Der Literaturbetrieb, vor allem die Literaturkritik, die Literaturgeschichtsschreibung und der Literaturunterricht, partizipieren an der Tradierung des Nationalgeistes. Die Literaturgeschichte zeichnet den Weg nach, den die Gründungsväter der nationalen Literatur gewiesen haben, und zeigt, wie die nachfolgenden Generationen diesen Weg fortzusetzen haben. Im 19. Jahrhundert hatte für Gervinus die Literaturgeschichte, und zwar mehr noch als die Politikgeschichte, die Aufgabe, das sich konstituierende Nationalbewusstsein herauszuarbeiten und daraus die Verpflichtung der Zeitgenossen auf den Prozess der historischen Verwirklichung der Nation abzuleiten. ${ }^{3}$

Die Literatur insistiert aber auch gern, vor allem seit der literarischen Moderne, auf ihrer Autonomie. Die Eigenart des Ästhetischen wird gerade in seiner Alterität zu kulturellen Werten und somit zur Nation gesehen.

\section{DIE MODERNE, DIE ENTWURZELUNGSERFAHRUNG UND VERSUCHE DER ENTNATIONALISIERUNG DER LITERATUR}

Kafka war einer der Protagonisten der literarischen Moderne. Deshalb reihte sich seine eigene literarische Produktion weder in die große noch in die kleine Literatur ein. Er schrieb nicht im Sinne der jüdischen Literatur, wie sie in Warschau funktionierte und die er als kleine Literatur charakterisierte. Er schrieb auch nicht nach der Tradition Goethes. Der Status des Fremden und Marginalisierten, den er als Jude hatte, und seine gleichzeitige Zugehörigkeit zur deutschen Kultur, die er genau kannte, verliehen ihm eine Freiheit und eine Fähigkeit zur Distanz und zur Verfremdung, die nicht alle hatten. Er konnte umso selbstverständlicher Positionen einnehmen, die andere, nicht-jüdische deutsche Schriftsteller nur mit großer Anstrengung erreichen konnten, weil sie für sie Bruch und Ablehnung der durch ihre Sozialisation verinnerlichten Werte und Denkmuster darstellten.

Das Bewusstsein einer existenziellen Entfremdung, der Entwurzelung, der Unmöglichkeit, an einer geteilten Wirklichkeit teilzuhaben, das Gefühl der Enge, all das drückten die avantgardistischen Schriftsteller aus. Für die Juden war dies erlebter Alltag. Kafkas Status als Jude brachte ihn dazu, eine Haltung zu entwickeln,

${ }^{3}$ Georg Gottfried Gervinus, Geschichte der poetischen National-Literatur der Deutschen, 5 Bde., 1835-1842 (Leipzig: Engelmann, 1846). 
die nicht mehr eine rein jüdische Haltung darstellt, sondern dem Gefühl einer ganzen Generation von Deutschen entspricht, die - aus denselben Gründen wie die Juden oder aus anderen Gründen - dasselbe Unbehagen erleben wie sie. Aus dieser Haltung hat sich eine neue Vorstellung von Kunst herauskristallisiert, die jedem Versuch, sie zu vereinnahmen, Widerstand leistet. Gerade gegenüber der Nation als Anrufungsinstanz zeigt sich diese Kunstvorstellung kritisch. Die Behauptung der Autonomie der Kunst stellt einen Versuch dar, ihre Indienstnahme durch andere Felder, vor allem durch die Politik, zu verweigern.

Der Eintritt einer politisch, sozial und kulturell marginalisierten Gruppe in das literarische Feld hat also entscheidend dazu beigetragen, dieses Feld grundlegend zu verändern, neu zu strukturieren und ein Gegenmodell zu traditionellen Werten und zu Legitimationsinstanzen zu erarbeiten. ${ }^{4}$

Diese ästhetische Revolution erlebten nationalistische Kreise in Deutschland als einen Angriff auf heilige Werte, die sie verehrten, und zwar auf die Nation, die bestehende soziale und politische Ordnung sowie auf die Tradition. Die Revolution in der Schreibart, die Dekonstruktion der Kunst als Institution, die Einführung des Grotesken, des Hässlichen, des Abnormalen, des Irrationalen, des Unbewussten, des Maßlosen als ästhetische Kategorien durch Bewegungen wie den Naturalismus, den Futurismus, den Dadaismus, den Surrealismus und den Expressionismus wurden als antinationale, pazifistische, weltbürgerliche und kosmopolitische Bestrebungen bekämpft. Die literarische und künstlerische Moderne wurde also von konservativen Kräften als jüdische Bewegung betrachtet. Und für sie bedeutete dies, dass diese Bewegung antieuropäisch und antideutsch war. ${ }^{5}$

Dies hat Konsequenzen für die Literaturwissenschaftspraxis in Deutschland, aber auch in ganz Europa gehabt. Der durch die Moderne eingeschlagene Weg der Entortung der Literatur wurde nie konsequent verfolgt. Auch wenn das Prinzip der Autonomie immer wieder proklamiert wird, bleibt die Literaturwissenschaft weitgehend nationalphilologisch orientiert. Einige Ansätze aber versuchen, diese Tendenz zu überwinden. Hier soll nun auf einige dieser Ansätze eingegangen werden.

\section{INTERKULTURELLE LITERATURWISSENSCHAFT}

Der Begriff interkulturelle Literaturwissenschaft hat sich inzwischen als Grundlage der Profilierung einer neuen Disziplin oder einer neuen wissenschaftlichen Praxis auch in Deutschland durchgesetzt, nachdem die interkulturelle Literaturwissenschaft vor allem in den USA entwickelt worden war. Dabei stützen sich die Wissenschaftler auf sehr unterschiedliche historische, fachliche

\footnotetext{
${ }^{4}$ Vgl. Konrad Kwiet, Gunther E. Grimm, Hans Peter Bayerdörfer, „Einleitung," in Im Zeichen Hiobs. Jüdische Schriftsteller und deutsche Literatur im 20. Jahrhundert, ed. Gunther E. Grimm, Hans Peter Bayerdörfer (Königstein/Ts: Verlag Athenäum, 1985).

${ }^{5}$ Vgl., ibid.
} 
und theoretische Traditionen und Erfahrungen und erarbeiten literaturhistorische und Textanalysemodelle, die stark divergierende Akzente setzen.

Mit der Interkulturalität als Kategorie der Literaturwissenschaft wird aber die traditionelle nationalphilologisch orientierte Literaturwissenschaft allgemein infrage gestellt. Literarische Texte werden nicht mehr als Ausdruck einer als homogen gedachten nationalen Kultur gelesen. Rekonstruiert werden nicht Kontinuität und Einheitlichkeit, Reinheit und Identität, Telos und Ethos, Wahrhaftigkeit und Genialität, Repräsentativität und Affirmation, Stimmigkeit und Totalität etc. Vielmehr werden alle diese Kategorien als Instrumentarien einer exegetischen Praxis betrachtet und behandelt, die sich selbst als kulturelle Gedächtnisarbeit in den Dienst einer wie auch immer verstandenen Perpetuierung oder gar Erneuerung der Nationalkultur stellt. Sie werden also als Symptome eines nationalistischen Bewusstseins historisiert und dekonstruiert. Die Grenzen einer traditionell nationalphilologischen Behandlung der Kultur hat der belgische Literaturwissenschaftler Marc Quaghebeur gerade an ihrer Unfähigkeit, die belgische Literatur und Kultur anders als in negativen Begriffen $\mathrm{zu}$ erfassen, demonstriert. An französischen Ansätzen analysiert er die Aporien gewohnter Kategorien und postuliert die Notwendigkeit, aber auch die Schwierigkeit, diesen kategorialen Rahmen zu überwinden und eine neue Sprache zu erfinden, die eine polyphone, polyglotte Literatur und Kultur wie die belgische, aber auch die schweizerische, zu erfassen vermag.

La première question que pose la "culture belge" - et les cultures de Belgique -, c'est précisément cette question du droit à une autre histoire et donc, à un autre type d'identité. Ce qu'elle pose en outre comme question, c'est celle de savoir comment la dire, comment la dire en Europe aujourd'hui, et qui plus est, comment la dire en français. Il s'agit en effet de savoir [...] comment écrire cela en français moderne, c'est-àdire dans la langue dont le destin historique s'est identifié (et dont la vertu intrinsèque découle de) la construction de l'État-nation le plus centralisé du monde. ${ }^{6}$

(Die erste Frage, die die ",belgische Kultur" - und die Kulturen von Belgien - aufwirft, betrifft eben das Recht auf eine andere Geschichte und somit auf einen anderen Typus von Identität. Eine Frage, die sie außerdem stellt, betrifft die Art und Weise, wie man diese ausdrücken kann und zwar heute in Europa und, was noch komplizierter ist, in der französischen Sprache. Es geht in der Tat darum zu wissen, [...] wie man dies in der modernen französischen Sprache schreiben kann, das heißt in der Sprache, deren historisches Schicksal darin bestanden hat (und deren intrinsische Tugend sich daraus ableitet), die zentralisierteste Staatsnation der Welt zu bilden.) [Übersetzung des Autors]

Die interkulturelle Literaturwissenschaft versucht, solche Aporien zu überwinden.

\footnotetext{
${ }^{6}$ Marc Quaghebeur, „Culture(s) belge(s), identité(s) et société," in Cahiers francophones d'Europe centreorientale 5-6 (1995): 175.
} 
Mit der Interkulturalität als Kategorie der Literaturwissenschaft wird nicht nur Distanz, sondern auch Nähe hergestellt. Und zwar die Nähe zum poststrukturalistischen Gestus der Überwindung westlicher metaphysischhumanistischer Entwürfe, Nähe zur Diskursanalyse und ihrer Aufdeckung von Machtdispositiven, Machtverhältnissen und Strategien, zur postmodernen Geographie und ihrer Auffassung von Raum, zum symbolischen Interaktionismus, zur postkolonialen Theorie und Kritik etc. Aus diesen Ansätzen übernimmt die interkulturelle Literaturwissenschaft Kategorien und Denkmuster, mit denen Literaturgeschichte neu geschrieben werden kann, mit denen klassische und neuere Texte nach ihrem Verhältnis $\mathrm{zu}$ transnationalen und transkulturellen historischen Wirklichkeiten, Herausforderungen und Bestrebungen, zu interkulturellen Spannungen, Konflikten und Rivalitäten befragt werden. Befragt werden sie auch nach den Mitteln, mit denen sie in solchen Spannungskontexten alte Kulturmuster festzuschreiben versuchen oder neue kulturelle Paradigmen aushandeln.

Gerade in Bezug auf Literaturgeschichte hat sich die nationalphilologische Perspektive in der Literaturwissenschaft durchgesetzt. Die Literaturgeschichte ist weitgehend die Geschichte der Literatur von Nationen und zielt darauf ab, die Literatur als Ort der Manifestation eines nationalen Geistes zu erfassen, der sich zielstrebig durch eine interne Gesetzmäßigkeit zu immer höheren Leistungen entwickelt oder auch Höhepunkte und Tiefschläge erlebt. Die Entwicklung wird in jedem Fall auf interne soziale, wirtschaftliche und geistige Triebkräfte zurückgeführt. Eine solche Literaturgeschichte gründet auf einer Auffassung von Nation als primordiale Kulturgemeinschaft, die Denk-, Gefühls- und Erfahrungshorizonte festlegt und somit Verhaltensmuster aller Mitglieder vereinheitlicht und festschreibt.

Auch traditionelle komparatistische Ansätze betonen zwar das Moment des Kontakts und des Austausches, bleiben aber weitgehend diesem national-kulturellen Modell verpflichtet. Diesbezüglich schreibt Michael Böhler:

\begin{abstract}
Vorherrschend in der Theorie der transkulturellen Beziehungen und Phänomene war bis vor Kurzem eine am nationalstaatlichen Paradigma des 19. Jahrhunderts und der Idee einer Nationalkultur bzw. Nationalliteratur orientierte Vorstellung. Kulturelle Beziehungen und Austauschprozesse finden hier zwischen den Nationen oder zwischen Individuen qua nationale Repräsentationsfiguren statt. Nationale Kulturen werden dabei als in sich geschlossene, einheitliche Ensembles symbolhafter Handlungen und Riten, von Texten und Diskursen, Gegenständen und Begriffen verstanden, womit sich eine Gemeinschaft über sich selbst und andere verständigt. Dem Einheits- und Geschlossenheitsdenken dieses nationalen und Kulturbegriffs entsprechend stehen in einem solchen Modell kulturelle und literarische Austauschprozesse in einem Zusammenhang mit nationalen Identitätskonstrukten und werden vor allem unter dem Aspekt des Eigenen und Fremden bzw. der aneignenden Eingrenzung oder Ausgrenzung und der assimilierenden Homogenisierung konzeptualisiert. ${ }^{7}$
\end{abstract}

\footnotetext{
${ }^{7}$ Michael Böhler, „National-Literatur will jetzt nicht viel sagen, die Epoche der Weltliteratur ist an der Zeit, und jeder muss jetzt dazu wirken, diese Epoche zu beschleunigen," Überlegungen zu den
} 


\section{EUROPA IN BEWEGUNG}

Der Postdamer Romanist Ottmar Ette hat kürzlich versucht, eine Theorie der europäischen Literatur jenseits gewohnter Territorialisierungen zu entwerfen. Ganz konsequent versucht er die vorherrschende Tradition, die den Werken einen bestimmten Wohnsitz zuschreibt und daher die europäische Literatur nur als additive Strukturen denken kann, zu überwinden. Er schlägt eine transversale Sichtweise der Literatur Europas vor, die deutlich machen soll, dass diese aus Werken besteht, die zwischen Innerhalb- und Außerhalbbefindlichkeit oszillieren. "Gerade die Literaturen ohne festen Wohnsitz, [...] verdeutlichen, in welchem Maße bei der Frage nach der europäischen Literatur eine Beschränkung auf die nationale oder die europäische Ebene obsolet geworden ist." ${ }^{18}$ Ette muss jedoch feststellen, dass seine Idee, Europa in Bewegung und Europa als Bewegung zu denken, vorerst nur eine prospektive, zukunftsgerichtete Vision bleiben wird, zumal die europäische Literarturwissenschaft weiterhin mit Kategorien aus dem 19. Jahrhundert arbeitet. Es mehren sich aber Versuche, die europäische Literatur im Kontext der Globalisierung als eine Weltliteratur zu denken, die den Prozess der Globalisierung widerspiegelt und neue Räume produziert, die nicht national und nicht einmal kontinental gedacht werden können. So schreibt der spanische Autor Juan Goytisolo in der Besprechung von Emine Sevgi Özdamars Werk „Das Leben ist eine Karawanserei, hat zwei Türen, aus einer kam ich rein, aus der anderen ging ich raus:"

Quite a long time ago, at a meeting of the French Institute in Madrid, I surprised the audience by coming out with the idea, that the future of French literature lay with writers from the Maghreb and Caribbean, English Literature with Pakistani and Hindu writers and German literature with Turks. My boutade was greeted with laughter $[\ldots]$ Now the joke is for real. ${ }^{9}$

Damit wären wir wieder bei der Situation angelangt, die zur Entstehung der Moderne und zum Versuch der Entnationalisierung der Literatur Ende des 19. Jahrhunderts führte. Wenn Nationen immer mehr Probleme mit der Inbesitznahme der Literatur haben, weil sich diese ihren gewohnten Kategorien entziehen, wenn Autoren aufgrund ihrer Biographie nicht mehr automatisch einer Volksgemeinschaft zugerechnet werden können, werden Kritiker und Wissenschaftler notwendigerweise neue Kategorien erfinden müssen, mit denen die Literatur geortet, geordnet und erfasst werden kann. Gerade solche Kategorien schlägt Ottmar Ette vor.

David Simo

dsimo@uycdc.uninet.cm

kulturtopographischen Raumstrukturen in der Gegenwartsliteratur. http://www.Goethezeitportal.de 8 Ottmar Ette, „Europäische Literatur(en) im globalen Kontext. Literaturen für Europa,“ in Wider den Kulturzwang. Migration, Kulturalisierung und Weltiteratur, hg. Özkan Ezli, Dorothee Kimmich und Annette Werberger (Bielefeld: transcript, 2009), 292.

9 Juan Goytisolo, Rezension: Life is a Caravansaray von Emine Sevgi Özdamar, Times Literary Supplement, 2. Dezember 1994, 12. 